\title{
Prediction of Melt Flow and Spread of Thermoplastic Objects with the Particle Finite Element Method
}

\author{
EUGENIO OÑATE, RICCARDO ROSSI, and SERGIO R. IDELSOHN \\ International Center for Numerical methods in Engineering (CIMNE) \\ Technical University of Catalonia (UPC) \\ Gran Capitán s/n, 08034 Barcelona, Spain \\ onate@cimne.upc.edu, www.cimne.com
}

\begin{abstract}
A new computational procedure for analysis of the melting and spread of polymers under fire conditions is presented. The method, termed Particle Finite Element Method (PFEM), combines concepts from particlebased techniques with those of the standard finite element method (FEM). The key feature of the PFEM is the use of an updated Lagrangian description to model the motion of nodes (particles) in the thermoplastic material. Nodes are viewed as material points which can freely move and even separate from the main analysis domain representing, for instance, the effect of melting and dripping of polymer particles. A mesh connects the nodes defining the discretized domain where the governing equations are solved as in the standard FEM. An incremental iterative scheme for the solution of the non linear transient coupled thermal-flow problem is used. Examples of the possibilities of the PFEM for the modelling and simulation of the melting and spread of polymers under different fire conditions are described. Numerical results are compared with experimental data provided by NIST.
\end{abstract}

KEYWORDS: melt flow, thermoplastic objects, melt spread, particle finite element method

\section{NOMENCLATURE LISTING}

\begin{tabular}{llll}
$v_{i}$ & velocity along ith axis & \multicolumn{2}{l}{ Greek } \\
$x_{j} \quad$ Coordinate along $j$ th axis & $\rho$ & density \\
$b_{i} \quad$ body force & $\sigma_{i j}$ & Cauchy stress component \\
$c \quad$ Specific heat & $\delta_{i j}$ & deviatoric stress \\
$p \quad$ pressure & $\mu$ & viscosity \\
$M, K, G, L, Q, C, H$ & $\phi$ & temperature \\
$\quad$ finite element matrices & $\pi_{i}$ & pressure gradient projection
\end{tabular}

$\tau_{i}$ stabilization parameter

$\varepsilon_{i j}$ strain rate component

\section{INTRODUCTION}

Thermoplastic objects, including mattresses, upholstered furniture, and molded objects such as electronic housings and automobile parts, respond to fire by melting and dripping onto the surface below. The flow of material affects heat and mass transport within the object, and the accumulating melt pool below the object extends the flaming zone and increases the overall rate of heat release [1,2]. If the fire from the object and the pool fire interact, the intensity of the fire is enhanced even further. The spread rate of the melt pool and its burning behavior (including whether it is even able to sustain ignition) are affected by the flooring material as well as by the properties of the melt.

Computer modeling and simulation of the melting, flow and flame spread of thermoplastics is extremely complex involving fluid flow, heat transfer, material degradation, flame chemistry, surface tension, and complex material properties [3]. In addition, the drastic changes in shape pose a severe challenge to traditional modeling methods. Attempts to model melt flow of polymeric material in fire using the volume of fluid (VOF) method have encountered difficulties with numerical instabilities and excessive runtimes [4].

The Particle Finite Element Method (PFEM, www.cimne.com/pfem) [5-11] presented in this work is a powerful technique for modelling and analysis of complex multidisciplinary problems in fluid mechanics involving coupled thermal effects, fragmentation and separation of fluid particles and fluid-structure interaction effects, among others. The PFEM combines ideas from Lagrangian particle-based techniques with the advantages of the integral formulation of the finite element method [15]. A key advantage of the Lagrangian formulation in the PFEM is the elimination of the convective terms in the fluid flow and 
thermal equations, which favours the simplicity (and symmetry) of the formulation, as well as computational efficiency.

In the PFEM, the particles represent the nodes of a finite element mesh. The particles can move freely according to the velocity field, transporting their momentum and physical properties. A robust and efficient remeshing algorithm connects the nodes into a finite element grid for solution of the state variables in the new configuration. The PFEM has been used to solve a variety of free surface, fluidstructure interaction, and multiphase problems, including dam bursting, breaking waves in harbours, ship hydrodynamics, and metal casting, among others [5,6,8-11].

This paper describes the key aspects of the PFEM for analysis of the melting, flow and flame spread of thermoplastic objects. The underlying ideas of the paper follow the original work of the authors in this field as presented in $[12,13]$. The recent developments of the PFEM described in this paper demonstrate the potential of this method for solving complex melting flow problems for two and three dimensional heated objects accounting for frictional contact and self-contact situations. The paper is structured as follows. In the next section the basis of the PFEM are summarized. The essential governing equations and an overview of the discretization procedure and the general solution scheme are given. The potential of the PFEM for simulation of the melt flow and spread of thermoplastic objects is shown in examples of application of melting of heated samples. Numerical results for one of the examples are compared with experimental results obtained at NIST [4].

\section{THE PARTICLE FINITE ELEMENT METHOD. AN OVERVIEW}

\section{Basic steps of the PFEM}

In the PFEM the analysis domain (which may include solid and fluid subdomains) is modeled with an updated Lagrangian formulation. That is, all variables in the fluid and solid domains are assumed to be known in the current configuration at time t. The new set of variables in both domains is sought for in the next or updated configuration at time $t+\Delta t$. The finite element method (FEM) is used to solve the continuum equations in both domains. Hence a mesh discretizing these domains must be generated in order to solve the governing equations for both the fluid and solid problems in the standard FEM fashion. To do this, the nodes discretizing the analysis domain are treated as material particles whose motion is tracked during the transient solution. This is useful to model the separation of particles from the main domain, such as in the case of dripping of melt particles in a thermoplastic object, and to follow their subsequent motion as individual particles with a known density, an initial acceleration and velocity and subject to gravity forces. Every node is a material point and hence is characterized by the density of the polymer material. The mass of a given domain is obtained by integrating the density at the different material points over the domain. The quality of the numerical solution depends on the discretization chosen as in the standard FEM. Adaptive mesh refinement techniques can be used to improve the solution in zones where large motions of the fluid or the structure occur.

For clarity purposes the collection or cloud of nodes pertaining to the analysis domain (containing fluid and solid domains) will be noted as $(C)$, the volume defining the analysis domain for the fluid and the solid as $(V)$, and the mesh discretizing both domains as $(M)$.

A typical solution with the PFEM involves the following steps.

1. The starting point at each time step is the cloud of points in the fluid and solid domains. For instance, ${ }^{n} C$ denotes the cloud at time $t=t_{n}$ (Fig. 1).

2. Identify the boundaries defining the analysis domain ${ }^{n} V$ in the fluid and the solid. This is an essential step as some boundaries (such as the free surface in fluids or in the melting of thermoplastic materials) may be severely distorted during the solution process including separation and re-entering of nodes. The Alpha-Shape method [14] is used for the boundary definition.

3. Discretize the fluid and solid domains with a finite element mesh ${ }^{n} M$. In this work an innovative mesh generation scheme based on the extended Delaunay tessellation has been used [7].

4. Solve the coupled Lagrangian equations of motion for the fluid and the solid domains. Compute the relevant state variables in both domains at the next (updated) configuration for $t+\Delta t$ : 
velocities, pressure, viscous stresses and temperature in the fluid and displacements, stresses, strains and temperature in the solid.

5. Move the mesh nodes to a new position ${ }^{n+1} C$ where $n+1$ denotes the time $t_{n}+\Delta t$, in terms of the time increment size. This step is typically a consequence of the solution process of step 4 .

6. Go back to step 1 and repeat the solution process for the next time step to obtain ${ }^{n+2} C$. The process is shown in Fig. 1.

A special feature of the PFEM is its ability to model frictional contact situations. The contact between two solid interfaces is simply treated by introducing a layer of contact elements between the two interacting solids. This layer is automatically created during the mesh generation step by prescribing a minimum distance $\left(h_{c}\right)$ between two solid boundaries. If the distance exceeds the minimum value $\left(h_{c}\right)$ then the generated elements are not taken into account, or else are treated as fluid air elements. Otherwise the elements are treated as contact elements where a relationship between the tangential and normal forces and the corresponding displacement is introduced so as to model elastic and frictional contact effects in the normal and tangential directions, respectively.

This algorithm has proven to be very effective and it allows to identifying and modeling complex frictional contact conditions between two or more interacting bodies moving in air or water in an extremely simple manner. Of course, the accuracy of this contact model depends on the critical distance $\left(h_{c}\right)$ above mentioned.

This contact algorithm can also be used effectively to model frictional contact conditions between rigid or elastic solids in standard structural mechanics applications. Examples of the performance of the frictional contact algorithm in the PFEM can be found in $[6,8,11]$.

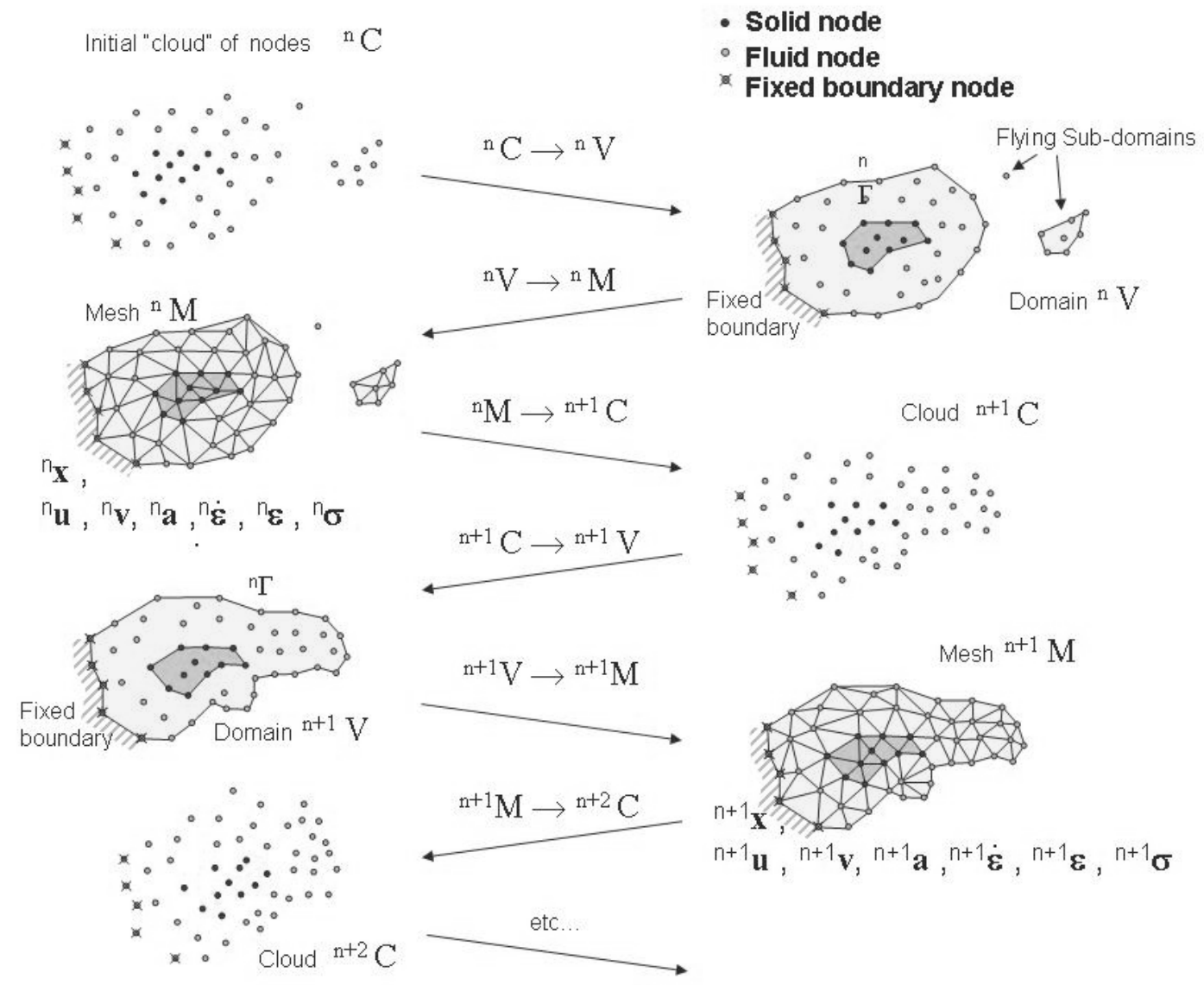

Fig. 1. Sequence of steps to update a "cloud" of nodes from time $n(t=t n)$ to time $n+2(t=t n+2 \Delta t)$. 


\section{Governing equations in the PFEM}

It is assumed in this work that the polymer melt flow is governed by the equations of an incompressible fluid with a temperature dependent viscosity. A quasi-rigid behaviour of the polymer object at room temperature is reproduced by using a very high value of the viscosity parameter. As temperature increases in the thermoplastic object due to heat exposure, the viscosity decreases in several orders of magnitude as a function of temperature and this induces the melt and flow of the particles in the heated zone. The key equations to be solved in the polymer melt flow problem, written in the Lagrangian frame of reference, are the following:

\section{Momentum}

$\rho \frac{\partial v_{i}}{\partial t}=\frac{\partial \sigma_{i j}}{\partial x_{j}}+b_{i} \quad$ in $\quad \Omega$

Mass balance

$\frac{\partial v_{i}}{\partial x_{i}}=0 \quad$ in $\quad \Omega$

Heat transport

$\rho c \frac{\partial \phi}{\partial t}=\frac{\partial}{\partial x_{i}}\left(k_{i} \frac{\partial \phi}{\partial x_{i}}\right)+Q \quad$ in $\quad \Omega$

In above equations $v_{i}$ is the velocity along the $i$ th global (cartesian) axis, $\phi$ is the temperature, $\rho, c$ and $k_{i}$ are the density (assumed constant), the specific heat and the conductivity of the material, respectively, $b_{i}$ and $Q$ are the body forces and the heat source, respectively and $\sigma_{i j}$ are the (Cauchy) stresses related to the rates of deformation by the standard constitutive equation (for incompressible material)

$\sigma_{i j}=s_{i j}-p \delta_{i j}$

$s_{i j}=2 \mu \varepsilon_{i j} \quad, \quad \varepsilon_{i j}=\frac{1}{2}\left(\frac{\partial v_{i}}{\partial x_{j}}+\frac{\partial v_{j}}{\partial x_{i}}\right)$

In Eqs. $4 s_{i j}$ is the deviatoric stresses, $\mathrm{p}$ is the pressure (assumed to be positive in compression), $\varepsilon_{i j}$ is the rate of deformation tensor and $\mu$ is the viscosity. In the following we will assume the viscosity $\mu$ to be a known function of temperature, i.e. $\mu=\mu(\phi)$. Modelling of the constitutive equation for the viscosity is performed via experimental laboratory tests. Indeed adopting a non-Newtonian rheology when the viscosity is a function of the strain rate and the pressure, in addition to the temperature is possible. This does not introduce any difficulty in the formulation nor in the numerical solution scheme, as the viscosity is evaluated at each iteration within a time step as a function of the state variables [10].

We note finally that all indexes in Eqs. $1-4$ range from $i, j=1, n_{d}$, where $n_{d}$ is the number of space dimensions of the problem (i.e. $n_{d}=2 \mathrm{D}$ for two-dimensional problems).

Eqs. 1-4 are completed with the standard boundary conditions of prescribed velocities and surface tractions in the mechanical problem and prescribed temperature and prescribed normal heat flux in the thermal problem. For details see $[6,8,9,10]$.

\section{Discretization of the equations}

The key problem in the numerical solution of Eqs. 1-4 is the satisfaction of the incompressibility condition (Eq. 2). A number of procedures to solve his problem exist in the finite element literature [11]. In our approach we use a stabilized formulation based in the so-called finite calculus procedure $[8,11,16]$. The essence of this method is the solution of a modified mass balance equation which is written as

$\frac{\partial v_{i}}{\partial x_{i}}+\sum_{i=1}^{3} \tau_{i}\left[\frac{\partial p}{\partial x_{i}}+\pi_{i}\right]=0$

where $\tau_{i}$ is a stabilization parameter given by [10] 
$\tau_{i}=\frac{3 h_{1}^{2}}{8 \mu}$

In above $h_{1}$ is a characteristic length of each finite element (such as $\left[A^{(e)}\right]^{1 / 2}$ for $2 \mathrm{D}$ elements). Also in Eq. 5 $\pi_{i}$ are auxiliary pressure projection variables chosen so as to ensure that the bracketed term in Eq. 5 vanishes for the exact solution [11]. This is ensured by adding the following constraint equation to the set of governing equations

$\frac{\partial p}{\partial x_{i}}+\pi_{i}=0 \quad i=1, n_{d}$

The integral equations are obtained by applying the standard Galerkin technique to the governing equations $1,3,5$ and 7 and the corresponding boundary conditions. For details see $[9,10,11,16]$.

We interpolate next in the standard finite element fashion set of governing variables, i.e. the velocities $v_{i}$, the pressure $p$, the temperature $\phi$ and the pressure gradient projections $\pi_{i}$. In our work we use equal order linear interpolation for all variables over meshes of 3-noded triangles (in 2D) and 4-noded tetrahedra (in 3D) $[7,11,15]$. The resulting set of discretized equations has the following form

\section{Momentum}

$\mathrm{M} \frac{\partial \overline{\mathrm{v}}}{\partial t}+\mathrm{K}(\mu) \bar{\phi}-\mathrm{G} \overline{\mathrm{p}}=\mathrm{f}_{1}$

Mass balance

$\mathrm{G}^{T} \overline{\mathrm{v}}+\mathrm{L} \overline{\mathrm{p}}+\mathrm{Q} \bar{\pi}=0$

\section{Pressure gradient projection}

$\hat{\mathrm{M}} \bar{\pi}+\mathrm{Q}^{T} \overline{\mathrm{p}}=0$

Heat transfer

$\mathrm{C} \frac{\partial \bar{\phi}}{\partial t}+\mathrm{H} \bar{\phi}=\mathrm{f}_{2}$

In Eqs. 8-11 $\overline{(\cdot)}$ denotes nodal variables. The definition of the different matrices and vectors in Eqs. 8-11 can be found in $[8,10,11]$. The solution in time of Eqs. 8-11 is performed using a fractional step procedure $[6,8,9,10,15]$. We note the coupling of the flow and thermal equations via the dependence of the viscosity $\mu$ with the temperature.

The effect of air surrounding the melting object is neglected in the analysis. Inclusion of this effect is indeed possible and it implies discretizing the air domain and solving for the motion of air particles with the PFEM $[9,10]$.

\section{ACCOUNTING FOR GASIFICATION EFFECTS}

The effect of gasification can be introduced approximately by adding a (nonlinear) energy loss term in the energy equation. This term represents the energy that migrates from the system to the gas due to the evaporation (gasification) of a part of the material. In mathematical terms this implies that the gasification heat flux has the following form

$q_{g a s}=A \varepsilon_{i i}, \quad \varepsilon_{i i}=\frac{\partial v_{i}}{\partial x_{i}}$

with

$\varepsilon_{i i}=f(\phi)$

where " $A$ " is an appropriate constant and $f(\phi)$ express the (generally non linear) relation between the volume variation and the temperature.

The idea followed in our work is rather simple: once the temperature field is known (obtained by solving over a fixed domain the non-linear heat equation 11), the volume variation $\varepsilon_{v}$ is also fixed at every point 
of the mesh. This is important as it allows to define a continuum distribution of the term $\varepsilon_{i i}$ on the whole fluid domain.

The computed mass loss has to be included in the fluid problem to ensure that the volume variation of the sample is correctly modeled. The approach is thus to solve the momentum equations prescribing as a constraint the local volume variation.

From a practical point of view this implies solving the modified system

$\frac{\partial v_{i}}{\partial x_{i}}=f(\phi)$

which can be achieved with a simple modification of the mass balance equation 2 . This introduces a new force term in the right hand side of Eq. 9.

Examples of the effect of gasification in the numerical results are currently under study by the authors.

\section{NUMERICAL EXAMPLES}

All problems have been solved with the PFIRE code developed by the authors group at CIMNE. PFIRE is an object oriented software environment where the PFEM formulation described in the paper has been implemented. Information from PFIRE can be obtained from the authors.

\section{Melting and flow of a rectangular slab}

In the first example shown the PFEM is used to simulate an experiment performed at NIST in which a slab of polymeric material is mounted vertically and exposed to uniform radiant heating on one face. Degradation of the polymer decreases its viscosity by several orders of magnitude and produces fuel gases. Polymer melt is captured by a pan below the sample.

A schematic of the apparatus used in the experiments is shown in Fig. 2. A rectangular polymeric sample of dimensions $10 \mathrm{~cm}$ high by $10 \mathrm{~cm}$ wide by $5 \mathrm{~cm}$ thick is mounted upright and exposed to uniform heating on one face from a radiant cone heater placed on its side. The sample is insulated on its lateral and rear faces. The melt flows down the heated face of the sample and drips onto a surface below. A load cell monitors the mass of polymer remaining in the sample, and a laboratory balance measures the mass of polymer falling onto the catch surface. More details of the experimental setup are given in previous publications $[4,12,13]$.

Fig. 2 shows all three curves of viscosity vs. temperature for the polypropylene type PP702N, a low viscosity commercial injection molding resin formulation. The relationship used in the model, as shown by the black line, connects the curve for the undegraded polymer to points A and B extrapolated from the viscosity curve for each melt sample to the temperature at which the sample was formed. The result is an empirical viscosity-temperature curve that implicitly accounts for molecular weight changes.

PP702N

Polymer Melt Apparatus

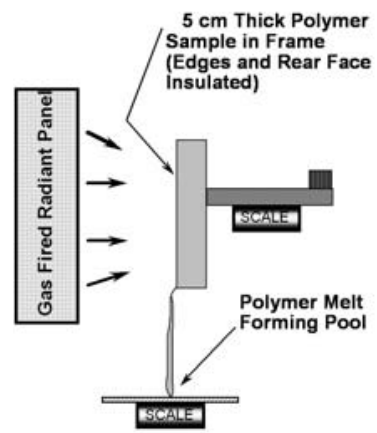

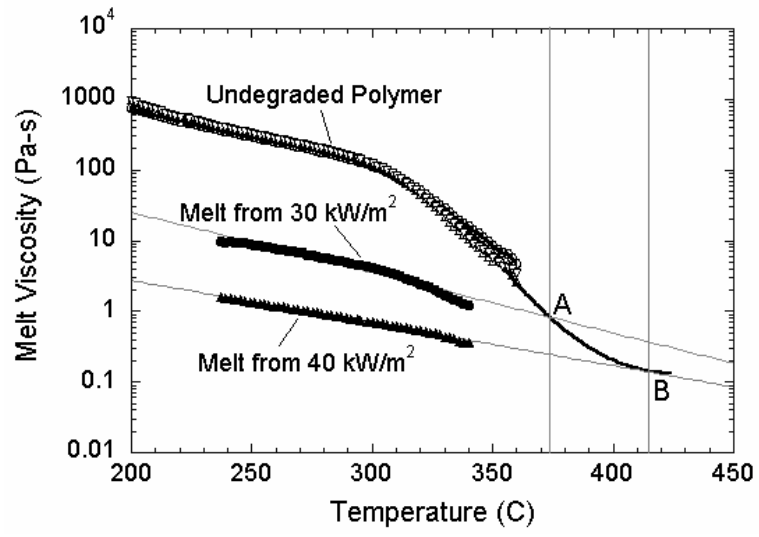

Fig. 2. Polymer melt experiment. Viscosity vs. temperature for PP702N polypropylene in its initial undegraded form and after exposure to $30 \mathrm{~kW} / \mathrm{m} 2$ and $40 \mathrm{~kW} / \mathrm{m} 2$ heat fluxes. The black curve follows the extrapolation of viscosity to high temperatures. 
An initial spacing of $2.0 \mathrm{~mm}$ between particles results in a two dimensional (2D) finite element model of 1537 nodes and 2818 elements, while a spacing of $1.4 \mathrm{~mm}$ results in 3098 nodes and 5832 elements. No particles are added during the course of the run.

The addition of a catch pan to capture the dripping polymer melt tests the ability of the PFEM model to recover mass when a particle or set of particles reaches the catch surface. For this problem, heat flux is only applied to free surfaces above the midpoint between the catch pan and the base of the sample. However, every free surface is subject to radiative and convective heat losses. To keep the melt fluid, the catch pan is set to a temperature of $600 \mathrm{~K}$. The thickness of the sample is reduced to $2.5 \mathrm{~cm}$ to achieve results more quickly. Indeed modelling of the solidification the melt in the catch pan is also possible with the present formulation. However, this has not been the objective of the present work.

Figure 3 shows four snapshots of the time evolution of the melt flow into the catch pan using the finer grid.
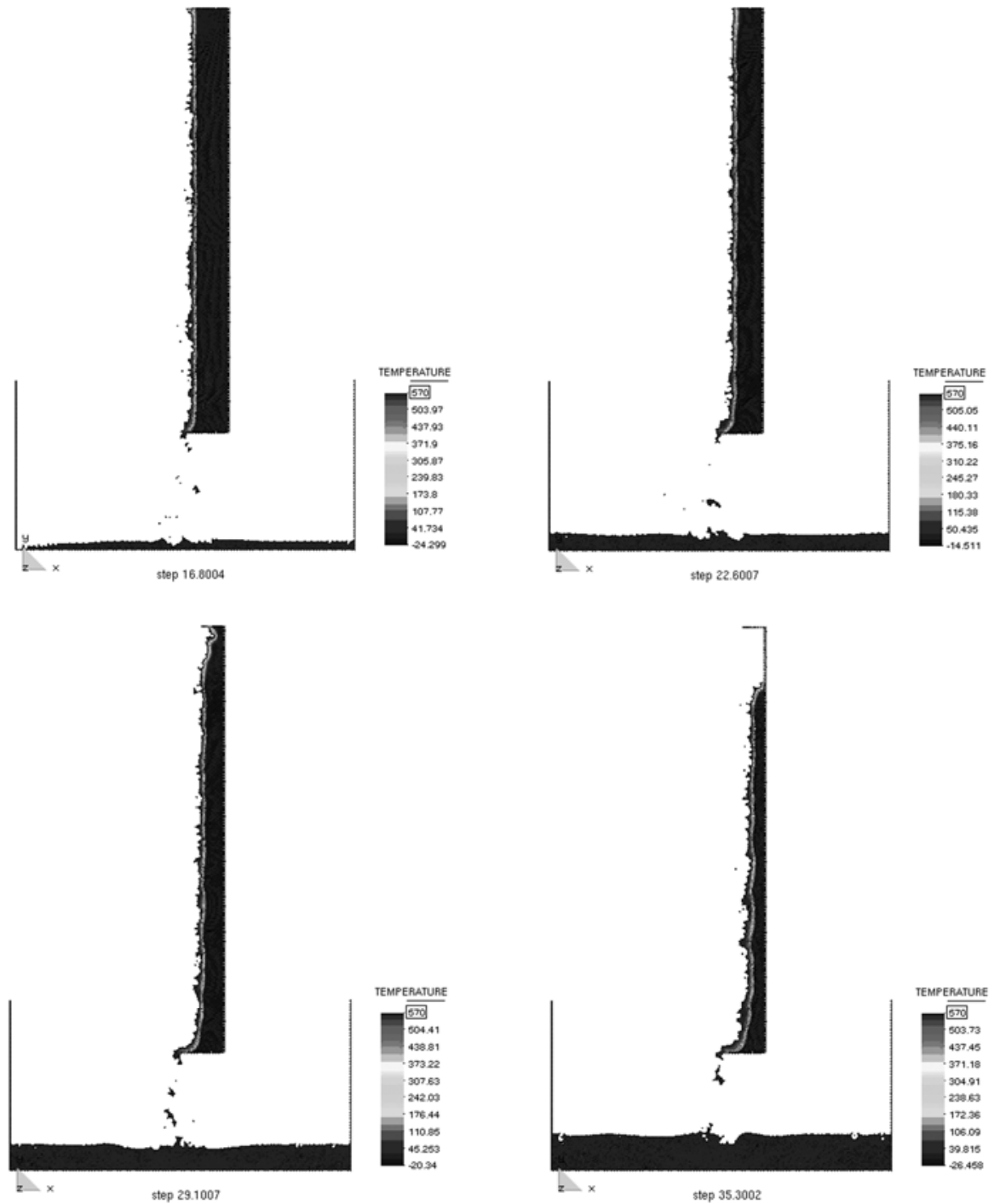

Fig. 3. Evolution of the melt flow into the catch pan at $t=400 \mathrm{~s}, 550 \mathrm{~s}, 700 \mathrm{~s}$ and $1000 \mathrm{~s}$.

Figure 4 (left) shows a detail of the flow into the catch pan at time $t=600 \mathrm{~s}$. Directly below the base of the sample where the melt is dripping, the temperature of the melt is higher. On the catch pan away from this point, the top of the melt has cooled to a temperature below the $600 \mathrm{~K}$ of the catch pan surface. The melt spreads to either side from the point at which the dripping melt contacts the catch pan. 
Figure 4 (right) shows the mass of the sample, the mass of the melt on the catch pan and their sum. After a heating time of about $170 \mathrm{~s}$, the mass begins to be transferred from the sample to the catch pan. The total mass reflects a conservation of mass within $\pm 5 \%$. This error can be reduced using a finer finite element discretization. Note that because of the way nodes are eliminated and recreated at surfaces via the Alpha Shape method in the PFEM, the total mass at times exceeds $100 \%$ of the initial mass.

In average however the Alpha Shape method preserves the total mass quite accurately, taking into account the complexity of the node elimination and recreation process.

Figure 5 compares the mass loss rate of the quasi-steady period with that obtained from experiments at three levels of heat flux. The mass loss rate follows the same trend, although the values are about $25 \%$ higher than the experimental data. Note that the model does not yet include gasification or in-depth absorption of radiation, both of which are expected to increase the mass loss rate. This discrepancy will eventually require further study.

The solution of the melt flow problem above described took some 3 CPU hours in a standard Pentium 4 PC.
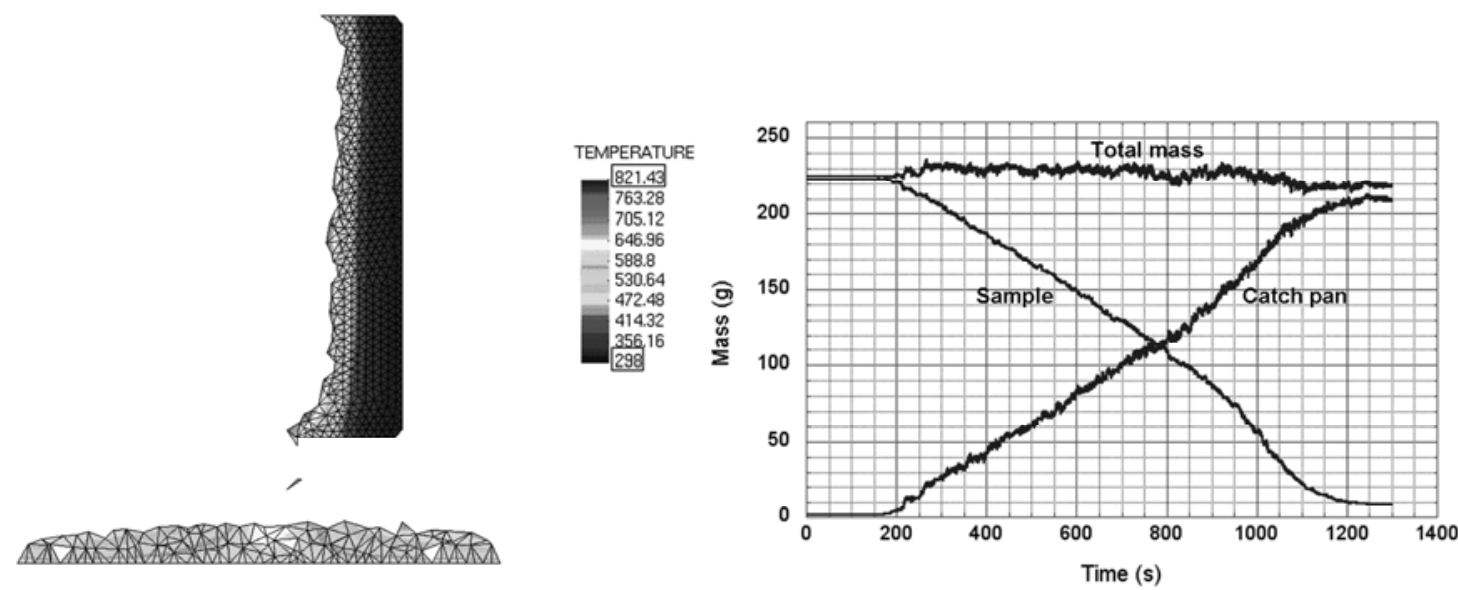

Fig. 4. Melt flow into catch pan at $t=600$ s. Mass vs time for polymer in sample, in catch pan and total mass.

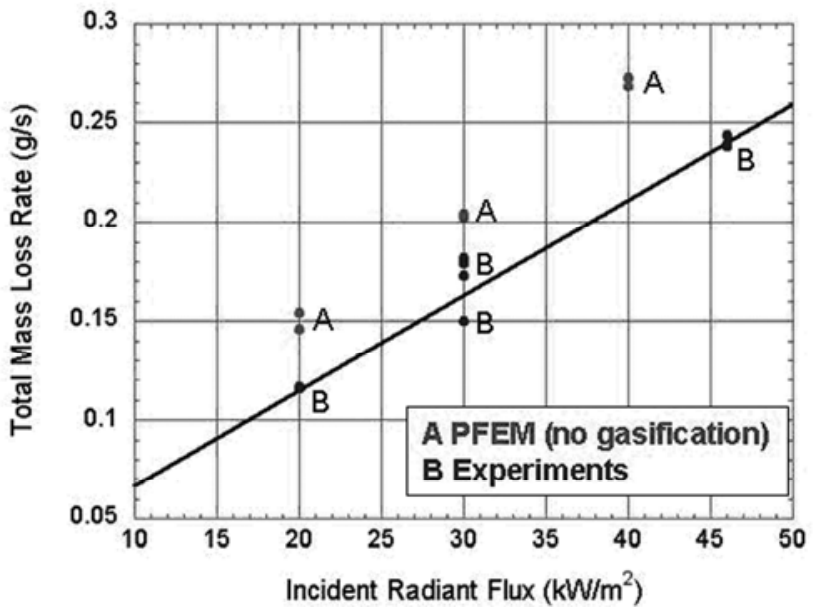

Fig. 5. Comparison of PFEM results to experiments for mass loss rate as a function of incident radiant flux.

To test the ability of the PFEM to solve this type of problem in three dimensions (3D), a 3D problem for flow from a heated sample was run. The same boundary conditions are used as in the 2D problem illustrated in Fig. 2 , but the initial dimensions of the sample are reduced to $10 \mathrm{~cm}$ high $2.5 \mathrm{~cm}$ wide and $2.5 \mathrm{~cm}$ thick. The initial size of the model is 22475 nodes and 97600 elements, and the runtime for this problem was 10.5 hours in a Pentium 4 PC. The shape of the surface and temperature field at different times after heating begins are shown in Fig. 6. 
Edge effects in the 3D model slow the rate of flow along the side walls, resulting in a thicker sample there throughout the melt flow process. This has also been observed in the experiments. Although the resolution for this problem is not fine enough to achieve high accuracy, the qualitative agreement of this 3D model with 2D flow and the ability to carry out this problem in a reasonable amount of time suggest that the PFEM can be used to model complex 3D flow geometries. Improved efficiency is however needed to make such calculations routine. The short term aim is to be able to solve $3 \mathrm{D}$ melt problems involving one million elements overnight.



Fig. 6. 3D melt flow from a heated prismatic sample at different times using the PFEM.

\section{Melting and flow of a triangular slab}

Figures 7 and 8 show results for the analysis of the melting of a triangular thermoplastic object. The material properties for the polymer are the same as for the previous example. The potential of the PFEM for simulating the progressive detachment and flow of the polymer particles from the object surface towards the underlying floor is clearly demonstrated in this example. A similar but more complex analysis is shown in Fig. 7. Here the problem is the simulation of the melt flow of a triangular thermoplastic object into a catch pan. The PFEM succeeds to predicting in a very realistic manner the progressive melting and slip of the polymer particles along the vertical wall separating the triangular object and the catch pan. The analysis follows until the whole object has fully melted and its mass is transferred to the catch pan. We note that the total mass was preserved with an accuracy of $0.5 \%$ in both these studies. Again gasification, in-depth absorption or radiation were not taken into account in these examples. 

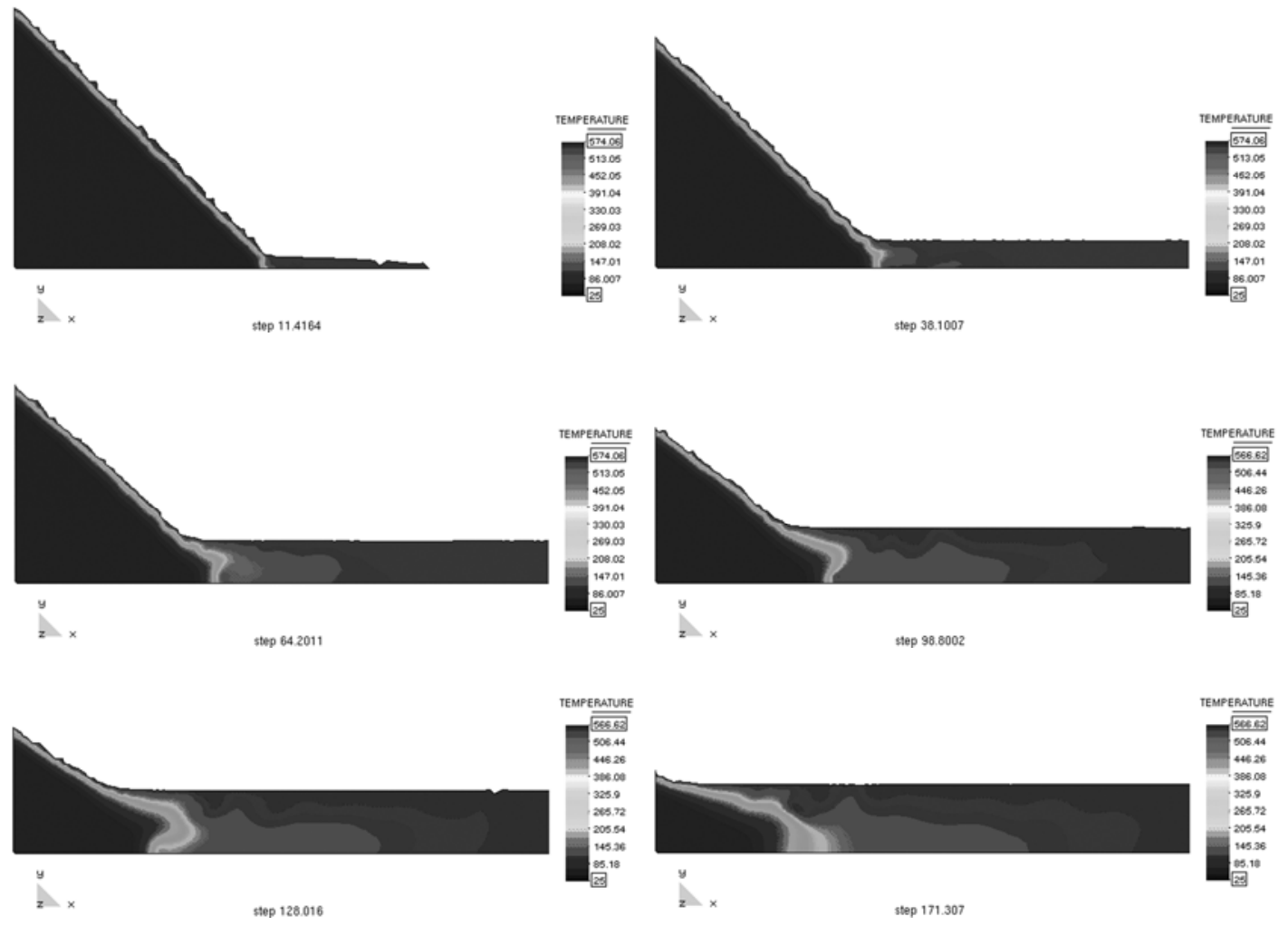

Fig. 7. Simulation of the melt flow of a heated triangular thermoplastic object.
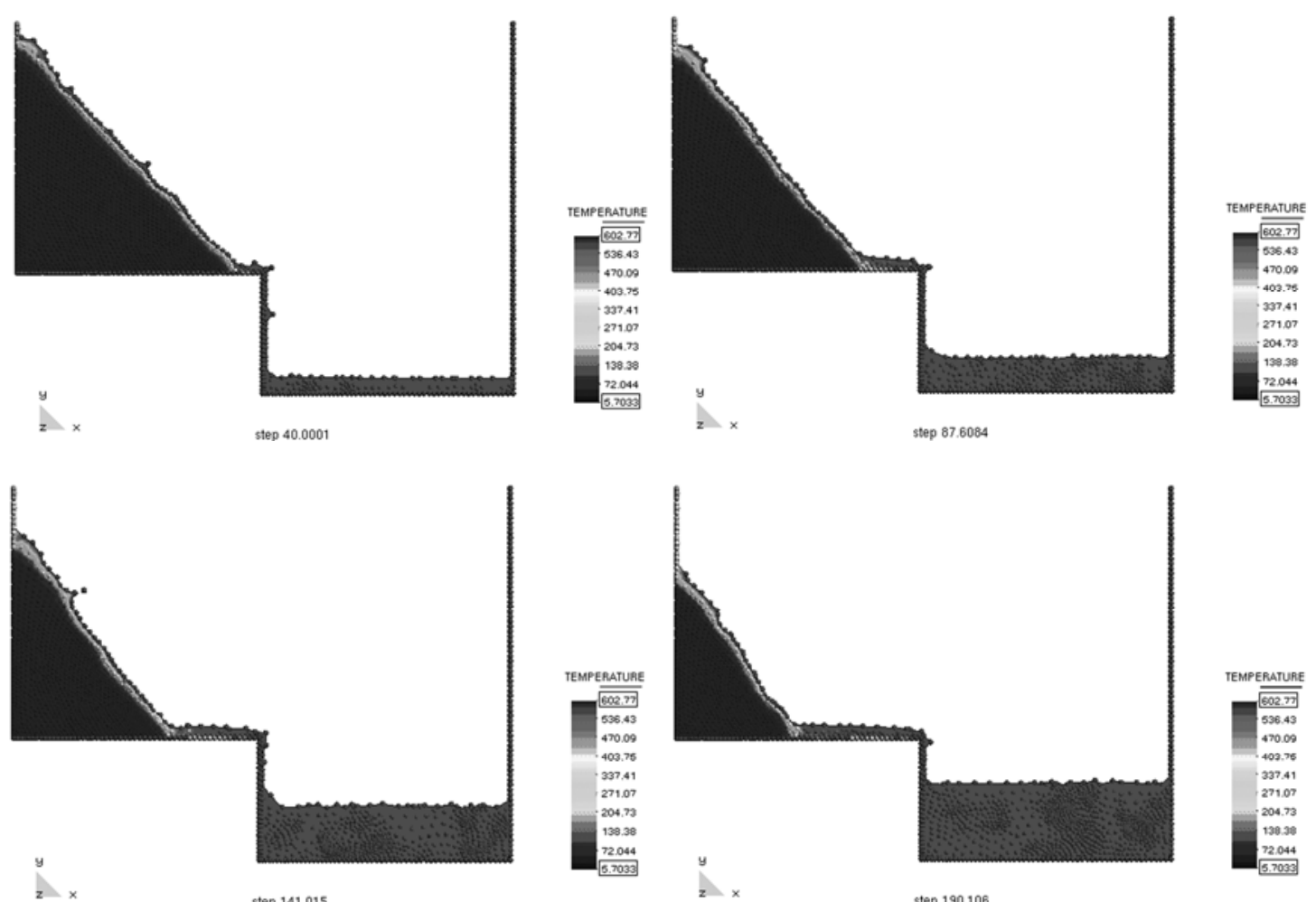
step 141.015 step 190.106 


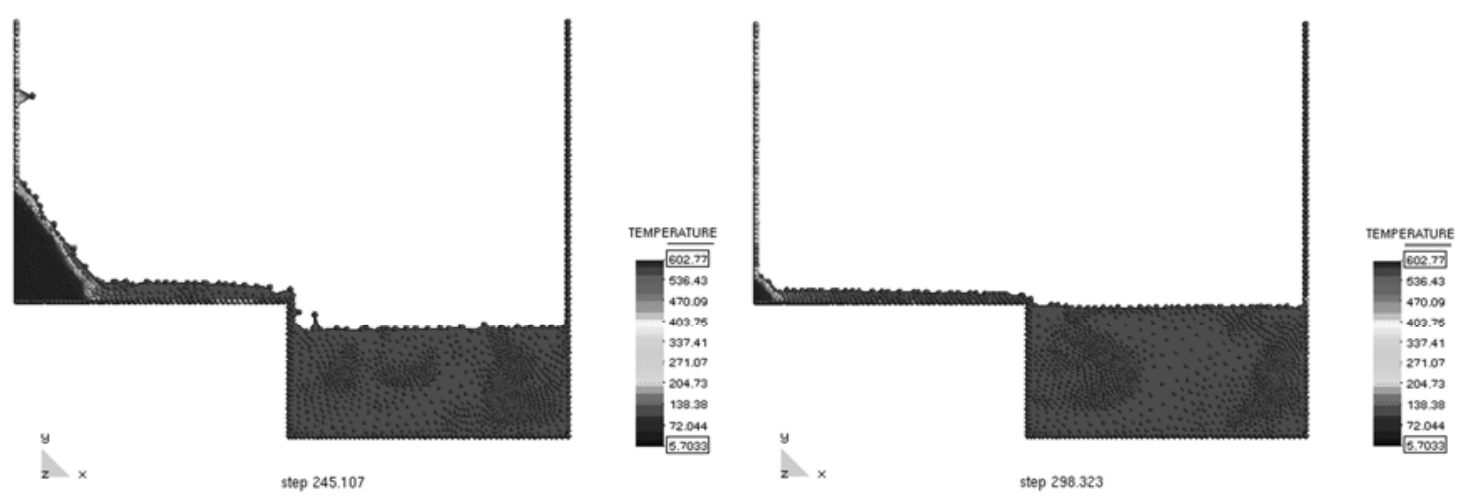

Fig. 8. Melt flow of a heated triangular object into a catch pan.

\section{Melting of a thermoplastic chair}

The last example shown in Fig. 9 is the simulation of the melt flow of a thermoplastic object ressembling a chair modeled as a 2D solid. The images show the progressive melting of the chair exposed to a heat flux of $30 \mathrm{kw} / \mathrm{m}^{2}$. The ability of the PFEM to model self-contact situations as the shape of the chair changes with time due to melting is noticeable.
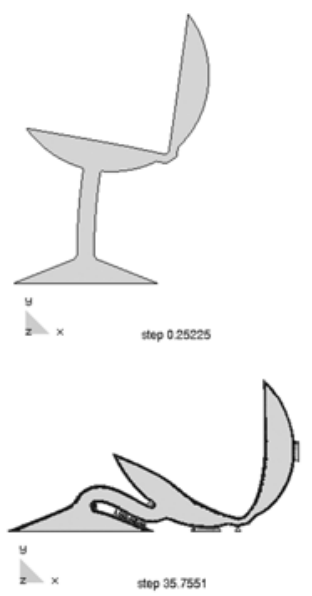
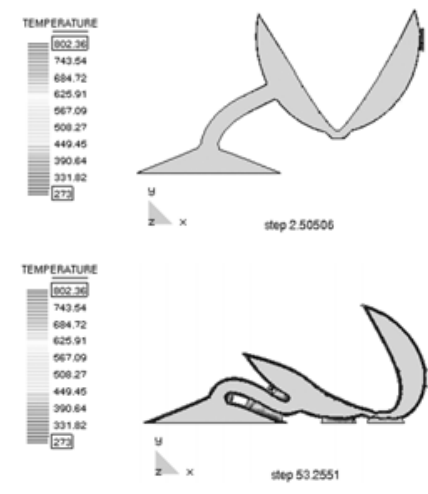
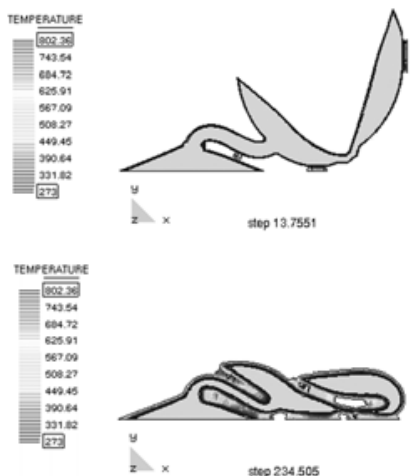
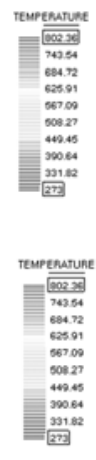

Fig. 9. Melting of a heated chair modeled as a 2D object. Note self contact between chair surface as melting evolves.

\section{CONCLUDING REMARKS}

The PFEM is a powerful technique to model the melting, flow and flame spread of thermoplastic objects in fire situations. The method allows to track the motion of the polymer particles as they melt, flow over the surface of the object and fall towards and on the underlying floor. The PFEM can also predict the spread of the melt in the floor for different ambient temperature conditions.

The simulation of the melting of a simplified chair of arbitrary shape has shown the potential of the PFEM to model the drastic change of shape of objects as they melt, including self-contact situations.

Further developments in progress include the study of gasification, in-depth absorption and radiation effects, coupling of the PFEM formulation with the external heat source, inclusion of elastic effects in the thermoplastic material and increasing the computational efficiency of the method. 


\section{ACKNOWLEDGMENT}

The authors thank NIST for providing experimental results for the 2D rectangular problem. Special thanks are given to Dr. K. Butler from NIST for her guidance and advice during this research.

\section{REFERENCES}

[1] Zhang, J., Shields, T.J., and Silcock, G.W.H., (1997) Effect of melting behaviour on flame spread of thermoplastics, Fire \& Materials 21(1): 1-6, Jan - Feb. doi:10.1002/(SICI)10991018(199701)21:1<1::AID-FAM583>3.0.CO;2-P

[2] Fleischmann, C.M. and Hill, G.R., (2004) Burning behaviour of upholstered furniture, Interflam 2004, pp. 907-916.

[3] Sherratt, J., and Drysdale, D., (2001) The effect of the melt-flow process on the fire behaviour of thermoplastics, Interflam 2001, pp. 149-159.

[4] Butler, K.M., Ohlemiller, T.J., and Linteris, G.T., (2004) A Progress Report on Numerical Modeling of Experimental Polymer Melt Flow Behavior, Interflam 2004, pp. 937-948.

[5] S Idelsohn, S.R., Oñate, E., and Del Pin F., (2003b) A lagrangian meshless finite element method applied to fluid-structure interaction problems, Computer and Structures 81: 655-671. doi:10.1016/S0045-7949(02)00477-7

[6] Idelsohn, S.R., Oñate, E., and Del Pin F., (2004) The particle finite element method: a powerful tool to solve incompressible flows with free-surfaces and breaking waves, Int. J. Num. Meth. Engng. 61: 964-989. doi:10.1002/nme.1096

[7] Idelsohn, S.R., Oñate, E., Calvo, N, and Del Pin F., (2003a) The meshless finite element method, Int. J. Num. Meth. Engng. 58(6): 893-912. doi:10.1002/nme.798

[8] Oñate, E., Idelsohn, S.R., Del Pin F., and Aubry, R., (2004) The particle finite element method. An overview, Int. J. Comput. Methods 1(2): 267-307. doi:10.1142/S0219876204000204

[9] Aubry, R., Idelsohn S.R., and Oñate E., (2005) Particle finite element method in fluid-mechanics including thermal convection-diffusion. Computers and Structures 83(17-18): 1459-1475. doi:10.1016/j.compstruc.2004.10.021

[10] Aubry, R., Idelsohn S.R., and Oñate E., (2006) Fractional step like schemes for free surface problems with thermal coupling using the Lagrangian PFEM. Computational Mechanics 38(4-5): 294-309. doi:10.1007/s00466-006-0058-5

[11] Oñate, E., Idelsohn, S.R., Celigueta, M.A., and Rossi, R., (2008) Advances in the particle finite element method for the analysis of fluid-multibody interaction and bed erosion in free surface flows. Accepted for publication in Comput. Meth. Appl. Mech. Engng. 197, (19-20), 1777-1800. doi:10.1016/j.cma.2007.06.005

[12] Butler, K.M., Oñate, E., Idelsohn, S.R., and Rossi, R., (2007) Modeling and simulation of the melting of polymers under fire conditions using the particle finite element method, $11^{\text {th }}$ Int. Fire Science \& Engineering Conference, University of London, Royal Halbway College, UK, 3-5 September.

[13] Rossi, R., Butler, K.M., Oñate, E., and Idelsohn, S.R., (2007) Modeling polymer melt flow using the Particle Finite Element Method. Advanced Research Workshop on Fire Computer Modeling, Gijón, Spain, October 18-20.

[14] Edelsbrunner, H., and Mucke, E.P., (1999) Three dimensional alpha shapes, ACM Trans. Graphics 13: 43-72. doi:10.1145/174462.156635

[15] Zienkiewicz, O.C., Taylor, R.L., and Nithiarasu, N. (2005) The finite element method. Vol. 3 Fluid Mechanics. Sixth Edition, Elsevier.

[16] Oñate E., (2004) Possibilities of finite calculus in computational mechanics. Int. Journal for Numerical Methods in Engineering 60(1): 255-281. doi:10.1002/nme.961 